\title{
Invariant and coinvariant spaces for the algebra of symmetric polynomials in non-commuting variables
}

\author{
François Bergeron and Aaron Lauve \\ LaCIM, Université du Québec à Montréal, CP 8888, Succ. Centre-ville, Montréal (Québec) H3C 3P8, CANADA
}

\begin{abstract}
We analyze the structure of the algebra $\mathbb{K}\langle\mathbf{x}\rangle^{\mathfrak{S}_{n}}$ of symmetric polynomials in non-commuting variables in so far as it relates to $\mathbb{K}[\mathbf{x}]^{\mathfrak{S}_{n}}$, its commutative counterpart. Using the "place-action" of the symmetric group, we are able to realize the latter as the invariant polynomials inside the former. We discover a tensor product decomposition of $\mathbb{K}\langle\mathbf{x}\rangle^{\mathfrak{S}_{n}}$ analogous to the classical theorems of Chevalley, Shephard-Todd on finite reflection groups. In the case $|\mathbf{x}|=\infty$, our techniques simplify to a form readily generalized to many other familiar pairs of combinatorial Hopf algebras.

Résumé. Nous analysons la structure de l'algèbre $\mathbb{K}\langle\mathbf{x}\rangle^{\mathfrak{S}_{n}}$ des polynômes symétriques en des variables non-commutatives pour obtenir des analogues des résultats classiques concernant la structure de l'anneau $\mathbb{K}[\mathbf{x}]^{\mathfrak{S}_{n}}$ des polynômes symétriques en des variables commutatives. Plus précisément, au moyen de "l'action par positions", on réalise $\mathbb{K}[\mathbf{x}]^{\mathfrak{S}_{n}}$ comme sous-module de $\mathbb{K}\langle\mathbf{x}\rangle^{\mathfrak{S}_{n}}$. On découvre alors une nouvelle décomposition de $\mathbb{K}\langle\mathbf{x}\rangle^{\mathfrak{S}_{n}}$ comme produit tensorial, obtenant ainsi un analogues des théorèmes classiques de Chevalley et Shephard-Todd. Dans le cas $|\mathbf{x}|=\infty$, nos techniques se simplifient en une forme aisément généralisables à beaucoup d'autres paires d'algèbres de Hopf familières.
\end{abstract}

Keywords: Chevalley theorem, symmetric group, noncommutative symmetric polynomials, set partitions

\section{Introduction}

One of the more striking results of the invariant theory of reflection groups is certainly the following: if $W$ is a finite group of $n \times n$ matrices, then there is a graded $W$-module decomposition of the polynomial $\operatorname{ring} S=\mathbb{K}[\mathbf{x}]$, in variables $\mathbf{x}=\left\{x_{1}, x_{2}, \ldots, x_{n}\right\}$, as a tensor product ${ }^{(\mathrm{i})}$

$$
S \simeq S_{W} \otimes S^{W}
$$

if and only if $W$ is a group generated by (pseudo) reflections. As usual, $S$ affords the natural $W$-module structure obtained by considering it as the symmetric space on the defining vector space $X^{*}$ for $W$, e.g.,

(i) We assume throughout that $\mathbb{K}$ is a field containing $\mathbb{Q}$.

1365-8050 (C) 2008 Discrete Mathematics and Theoretical Computer Science (DMTCS), Nancy, France 
$w \cdot f(\mathbf{x})=f(w \cdot \mathbf{x})$. It is customary to denote by $S^{W}$ the ring of $W$-invariant polynomials for this action. To finish parsing (1), recall that $S_{W}$ stands for the coinvariant space, i.e., the $W$-module defined as

$$
S_{W}:=S /\left\langle S_{+}^{W}\right\rangle,
$$

the quotient of $S$ by the ideal generated by constant-term free $W$-invariant polynomials. We give $S, S^{W}$, and $S_{W}$ a grading by polynomial degree in $\mathbf{x}$ (the latter being well-defined because $\left\langle S_{+}^{W}\right\rangle$ is a homogeneous ideal). The motivation behind the quotient in (2) is to eliminate redundant copies of irreducible $W$-modules inside $S$. Indeed, if $\mathcal{V}$ is such a module and $f(\mathbf{x})$ is any $W$-invariant polynomial with no constant term, then $\mathcal{V} f(\mathbf{x})$ is an isomorphic copy of $\mathcal{V}$ living within $\left\langle S_{+}^{W}\right\rangle$. As a result, the coinvariant space $S_{W}$ is the interesting part of the story.

The context for the present paper is the algebra $T=\mathbb{K}\langle\mathbf{x}\rangle$ of noncommutative polynomials, with $W$ module structure on $T$ obtained by considering it as the tensor space on the defining space $X^{*}$ for $W$. In the special case when $W$ is the symmetric group $\mathfrak{S}_{n}$, we elucidate a relationship between the space $S^{W}$ and the subalgebra $T^{W}$ of $W$-invariants in $T$. The subalgebra $T^{W}$ was first studied in $[14,5]$ with the aim of obtaining noncommutative analogs of classical results concerning symmetric function theory. Recent work in $[12,3]$ has extended a large part of the story surrounding $(1)$ to this noncommutative context. In particular, there is an explicit $\mathfrak{S}_{n}$-module decomposition of the form $T \simeq T_{\mathfrak{S}_{n}} \otimes T^{\mathfrak{S}_{n}}$, cf. [3, Theorem 8.7].

By contrast, our work proceeds in a somewhat complementary direction. We consider $\mathcal{N}=T^{\mathfrak{S}_{n}}$ as a tower of $\mathfrak{S}_{d}$-modules under the "place-action" and realize $S^{\mathfrak{S}_{n}}$ inside $\mathcal{N}$ as a subspace $\Lambda$ of invariants for this action. This leads to a decomposition of $\mathcal{N}$ analogous to (1). More explicitly, our main result is as follows.

Theorem 1 There is an explicitly constructed subspace $\mathcal{C}$ of $\mathcal{N}$ so that $\mathcal{C}$ and the place-action invariants $\Lambda$ exhibit a graded vector space isomorphism

$$
\mathcal{N} \simeq \mathcal{C} \otimes \Lambda .
$$

As an immediate corollary we derive the Hilbert series formula

$$
\operatorname{Hilb}_{t}(\mathcal{C})=\operatorname{Hilb}_{t}(\mathcal{N}) \prod_{i=1}^{n}\left(1-t^{i}\right)
$$

Here, as usual, the Hilbert series of a graded space $\mathcal{V}=\bigoplus_{d \geq 0} \mathcal{V}_{d}$ is the formal power series defined as

$$
\operatorname{Hilb}_{t}(\mathcal{V})=\sum_{d \geq 0} \operatorname{dim} \mathcal{V}_{d} t^{d}
$$

where $\mathcal{V}_{d}$ is the homogeneous degree $d$ component of $\mathcal{V}$. The fact that (4) expands as a series in $\mathbb{N} \llbracket t \rrbracket$ is not at all obvious, as one may check that the Hilbert series of $\mathcal{N}$ is

$$
\operatorname{Hilb}_{t}(\mathcal{N})=1+\sum_{k=1}^{n} \frac{t^{k}}{(1-t)(1-2 t) \cdots(1-k t)}
$$

(taking $n=|\mathbf{x}|$ ). We underline that the harder part of our work lies in working out the case $n<\infty$. This is accomplished in Section 6. If we restrict ourselves to the case $n=\infty$, both $\mathcal{N}$ and $\Lambda$ become Hopf 
algebras and things are much simpler. Our results are then consequences of a general theorem of Blattner, Cohen and Montgomery. As we will see in Section 5, stronger results hold in this simpler context. For example, (4) may be refined to a statement about "shape" enumeration.

\section{The algebra $S^{\mathfrak{S}}$ of symmetric polynomials}

\subsection{Vector space structure of $S^{\mathfrak{S}}$}

We specialize our introductory discussion to the group $W=\mathfrak{S}_{n}$ of permutation matrices. The action on $S=\mathbb{K}[\mathbf{x}]$ is simply the permutation action $\sigma \cdot x_{i}=x_{\sigma(i)}$ and $S^{\mathfrak{S}_{n}}$ comprises the usual symmetric polynomials. We suppress $n$ in the notation and denote the subring of symmetric polynomials by $S^{\mathfrak{S}}$. (Note that upon sending $n$ to $\infty$, the elements of $S^{\mathfrak{S}}$ become formal series in $\mathbb{K} \llbracket \mathbf{x} \rrbracket$ of bounded degree; we still call them polynomials to affect a uniform discussion.) A monomial in $S$ of degree $d$ may be written as follows: given an $r$-subset $\mathbf{y}=\left\{y_{1}, y_{2}, \ldots, y_{r}\right\}$ of $\mathbf{x}$ and a composition of $d$ into $r$ parts, $\boldsymbol{a}=\left(a_{1}, a_{2}, \ldots, a_{r}\right)\left(a_{i}>0\right)$, we write $\mathbf{y}^{\boldsymbol{a}}$ for $y_{1}^{a_{1}} y_{2}^{a_{2}} \cdots y_{r}^{a_{r}}$. We assume that the variables $y_{i}$ are naturally ordered, so that whenever $y_{i}=x_{j}$ and $y_{i+1}=x_{k}$ we have $j<k$. Reordering the entries of a composition $\boldsymbol{a}$ in decreasing order results in a partition $\lambda(\boldsymbol{a})$ called the shape of $\boldsymbol{a}$. Summing over monomials $\mathbf{y}^{\boldsymbol{a}}$ with the same shape leads to the monomial symmetric polynomial

$$
m_{\mu}=m_{\mu}(\mathbf{x}):=\sum_{\lambda(\boldsymbol{a})=\mu, \mathbf{y} \subseteq \mathbf{x}} \mathbf{y}^{\boldsymbol{a}} .
$$

Letting $\mu=\left(\mu_{1}, \ldots, \mu_{r}\right)$ run over all partitions of $d=|\mu|=\mu_{1}+\cdots+\mu_{r}$ gives a basis for $S_{d}^{\mathfrak{S}}$. As usual, we set $m_{0}:=1$ and agree that $m_{\mu}=0$ if $\mu$ has too many parts (i.e., $n<r$ ).

\subsection{Dimension enumeration}

A fundamental result in the invariant theory of $\mathfrak{S}_{n}$ is that $S^{\mathfrak{S}}$ is generated by a family $\left\{f_{k}\right\}_{1 \leq k \leq n}$ of algebraically independent symmetric polynomials, having respective degrees $\operatorname{deg} f_{k}=k$. (One may choose $\left\{m_{k}\right\}_{1 \leq k \leq n}$ for such a family.) It follows immediately that the Hilbert series of $S^{\mathfrak{S}}$ is

$$
\operatorname{Hilb}_{t}\left(S^{\mathfrak{S}}\right)=\prod_{i=1}^{n} \frac{1}{1-t^{i}} .
$$

Recalling that the Hilbert series of $S$ is $(1-t)^{-n}$, we see from (1) and (6) that the Hilbert series for the coinvariant space $S_{\mathfrak{S}}$ is the well-known $t$-analog of $n$ !:

$$
\prod_{i=1}^{n} \frac{1-t^{i}}{1-t}=\prod_{i=1}^{n}\left(1+t+\cdots+t^{i-1}\right) .
$$

In particular, contrary to the situation in (4), the series $\operatorname{Hilb}_{t}(S) / \operatorname{Hilb}_{t}\left(S^{\mathfrak{S}}\right)$ in $\mathbb{Z} \llbracket t \rrbracket$ is obviously positive.

\subsection{Algebra and coalgebra structures of $S^{\mathfrak{S}}$}

Given partitions $\mu$ and $\nu$, there is an explicit formula for computing the product $m_{\mu} \cdot m_{\nu}$. In lieu of giving the formula, we refer the reader to $[3, \S 4.1]$ and simply give an example:

$$
m_{21} \cdot m_{11}=3 m_{2111}+2 m_{221}+2 m_{311}+m_{32} .
$$


The extremal terms above are relevant to our coming discussion. Note that if $n<4$, then the first term disappears. However, if $n$ is sufficiently large then analogs of these terms always appear with positive integer coefficients for a given pair $(\mu, \nu)$. If $\mu=\left(\mu_{1}, \ldots, \mu_{r}\right)$ and $\nu=\left(\nu_{1}, \ldots, \nu_{s}\right)$ with $r \leq s$, then the partition indexing the left-most term is denoted by $\mu \cup \nu$ and is given by sorting the list $\left(\mu_{1}, \ldots, \mu_{r}, \nu_{1}, \ldots, \nu_{s}\right)$ in increasing order; the right-most term is indexed by $\mu+\nu:=\left(\mu_{1}+\nu_{1}, \ldots, \mu_{r}+\nu_{r}, \nu_{r+1}, \ldots, \nu_{s}\right)$. Taking $\mu=31$ and $\nu=221$, we would have $\mu \cup \nu=32211$ and $\mu+\nu=531$.

The ring $S^{\mathfrak{S}}$ is also afforded a coalgebra structure with coproduct $\Delta: S_{d}^{\mathfrak{S}} \rightarrow \bigoplus_{k=0}^{d} S_{k}^{\mathfrak{S}} \otimes S_{d-k}^{\mathfrak{S}}$ and counit $\varepsilon: S^{\mathfrak{S}} \rightarrow \mathbb{K}$ given, respectively, by

$$
\Delta\left(m_{\mu}\right)=\sum_{\theta \cup \nu=\mu} m_{\theta} \otimes m_{\nu} \quad \text { and } \quad \varepsilon\left(m_{\mu}\right)=\delta_{\mu, 0} .
$$

In the case $n=\infty, \Delta$ and $\varepsilon$ are algebra maps, making $S^{\mathfrak{S}}$ a connected graded (by degree) Hopf algebra.

\section{The algebra $\mathcal{N}$ of noncommutative symmetric polynomials}

\subsection{Vector space structure of $\mathcal{N}$}

Suppose now that $\mathbf{x}$ denotes a set of non-commuting variables. The algebra $T=\mathbb{K}\langle\mathbf{x}\rangle$ of noncommutative polynomials is graded by degree. A degree $d$ noncommutative monomial $\mathbf{z} \in T_{d}$ is simply a length- $d$ "word":

$$
\mathbf{z}=z_{1} z_{2} \cdots z_{d}, \quad \text { with each } \quad z_{i} \in \mathbf{x} .
$$

In other terms, $\mathbf{z}$ is a function $\mathbf{z}:[d] \rightarrow \mathbf{x}$, with $[d]$ denoting the set $\{1, \ldots, d\}$. The permutation-action on $\mathbf{x}$ clearly extends to $T$, giving rise to the subspace $\mathcal{N}=T^{\mathfrak{S}}$ of noncommutative $\mathfrak{S}$-invariants. With the aim of describing a linear basis for the homogeneous component $\mathcal{N}_{d}$, we next introduce set partitions of $[d]$ and the type of a monomial $\mathbf{z}:[d] \rightarrow \mathbf{x}$. We write $\mathbf{A} \vdash[d]$ when $\mathbf{A}=\left\{A_{1}, \ldots, A_{r}\right\}$ is a set partition of $[d]$, i.e., $A_{1} \cup \ldots \cup A_{r}=[d]$, with $A_{i} \neq \emptyset$ and $A_{i} \cap A_{j}=\emptyset$ whenever $i \neq j$. The type $\tau(\mathbf{z})$ of a degree $d$ monomial $\mathbf{z}:[d] \rightarrow \mathbf{x}$ is the set partition

$$
\tau(\mathbf{z}):=\left\{\mathbf{z}^{-1}(x) \mid x \in \mathbf{x}\right\} \backslash\{\emptyset\} \quad \text { of } \quad[d],
$$

whose parts are the non-empty fibers of the function $\mathbf{z}$. For instance,

$$
\tau\left(x_{1} x_{8} x_{1} x_{5} x_{8}\right)=\{\{1,3\},\{2,5\},\{4\}\} .
$$

In the sequel, we lighten the heavy notation for set partitions, writing, e.g., $\{\{1,3\},\{2,5\},\{4\}\}$ as 13.25.4. Clearly the type of a monomial is a finite set partition with at most $n$ parts. Note that we may always order the parts in increasing order of their minimum elements. The shape $\lambda(\mathbf{A})$ of a set partition $\mathbf{A}=\left\{A_{1}, \ldots, A_{r}\right\}$ is the (integer) partition $\lambda\left(\left|A_{1}\right|, \ldots,\left|A_{r}\right|\right)$ obtained by sorting the part sizes of $\mathbf{A}$ in increasing order. Observing that the permutation-action is type preserving, we are led to consider the monomial linear basis for the space $\mathcal{N}_{d}$ :

$$
m_{\mathbf{A}}=m_{\mathbf{A}}(\mathbf{x}):=\sum_{\tau(\mathbf{z})=\mathbf{A}} \mathbf{z}
$$

For example, with $n=2$, we have $m_{\emptyset}=1, m_{1}=x_{1}+x_{2}, m_{12}=x_{1}^{2}+x_{2}^{2}, m_{1.2}=x_{1} x_{2}+x_{2} x_{1}$, $m_{123}=x_{1}{ }^{3}+x_{2}{ }^{3}, m_{12.3}=x_{1}^{2} x_{2}+x_{2}{ }^{2} x_{1}, m_{13.2}=x_{1} x_{2} x_{1}+x_{2} x_{1} x_{2}, m_{1.23}=x_{1} x_{2}{ }^{2}+x_{2} x_{1}{ }^{2}$, $m_{1.2 .3}=0, \ldots$ (Note that we set $m_{\emptyset}:=1$, taking $\emptyset$ as the unique set partition of the empty set, and we agree that $m_{\mathbf{A}}=0$ if $\mathbf{A}$ is a set partition with more than $n$ parts.) 


\subsection{Dimension enumeration and shape grading}

Above, we determined that $\operatorname{dim} \mathcal{N}_{d}$ is the number of set partitions of $d$ into at most $n$ parts. These are counted by the (length restricted) Bell numbers $B_{d}^{(n)}$. Then (5) follows from the fact that its right-hand side is the ordinary generating function for length restricted Bell numbers. See [9, §2]. We next highlight a finer enumeration, where we grade $\mathcal{N}$ by shape rather than degree.

For each partition $\mu$, we may consider the submodule $\mathcal{N}_{\mu}$ spanned by those $m_{\mathbf{A}}$ for which $\lambda(\mathbf{A})=\mu$. This results in a direct sum decomposition $\mathcal{N}_{d}=\bigoplus_{\mu \vdash d} \mathcal{N}_{\mu}$. A simple dimension description for $\mathcal{N}_{d}$ takes the form of a shape Hilbert series in the following manner. View commuting variables $q_{i}$ as marking parts of size $i$ and set $\boldsymbol{q}_{\mu}:=q_{\mu_{1}} q_{\mu_{2}} \cdots q_{\mu_{r}}$. Then

$$
\operatorname{Hilb}_{\boldsymbol{q}}\left(\mathcal{N}_{d}\right)=\sum_{\mu \vdash d} \operatorname{dim} \mathcal{N}_{\mu} \boldsymbol{q}_{\mu},=\sum_{\mathbf{A} \vdash[d]} q_{\lambda(\mathbf{A})} .
$$

Here, $\boldsymbol{q}_{\mu}$ is a marker for set partitions of shape $\lambda(\mathbf{A})=\mu$ and the sum is over all partitions into at most $n$ parts. Such a shape grading also makes sense for $S_{d}^{\mathfrak{S}}$. Summing over all $d \geq 0$ and all $\mu$, we get

$$
\operatorname{Hilb}_{\boldsymbol{q}}\left(S^{\mathfrak{S}}\right)=\sum_{\mu} \boldsymbol{q}_{\mu}=\prod_{i \geq 1}^{n} \frac{1}{1-q_{i}} .
$$

Using classical combinatorial arguments (cf. Chapter 2.3 of [2], Example 13), we see that the enumerator polynomials $\mathrm{Hilb}_{\boldsymbol{q}}\left(\mathcal{N}_{d}\right)$ are naturally collected in the exponential generating function

$$
\sum_{d=0}^{\infty} \operatorname{Hilb}_{\boldsymbol{q}}\left(\mathcal{N}_{d}\right) \frac{t^{d}}{d !}=\sum_{m=0}^{n} \frac{1}{m !}\left(\sum_{k=1}^{\infty} q_{k} \frac{t^{k}}{k !}\right)^{m} .
$$

For example, with $n=3$, we have

$$
\operatorname{Hilb}_{\boldsymbol{q}}\left(\mathcal{N}_{6}\right)=q_{6}+6 q_{5} q_{1}+15 q_{4} q_{2}+15 q_{4} q_{1}^{2}+10 q_{3}^{2}+60 q_{3} q_{2} q_{1}+15 q_{2}^{3},
$$

thus $\operatorname{dim} \mathcal{N}_{222}=15$ when $n \geq 3$. Evidently, the $\boldsymbol{q}$-polynomials $\operatorname{Hilb}_{\boldsymbol{q}}\left(\mathcal{N}_{d}\right)$ specialize to the length restricted Bell numbers $B_{d}^{(n)}$ when we set all $q_{k}$ equal to 1 .

In view of (10), (11), and Theorem 1, we are led to claim the following refinement of (4).

Corollary 2 For $n=\infty$, the shape Hilbert series of the space $\mathcal{C}$ is given by the expression

$$
\operatorname{Hilb}_{\boldsymbol{q}}(\mathcal{C})=\left.\sum_{d \geq 0} d ! \exp \left(\sum_{k=1}^{\infty} q_{k} \frac{t^{k}}{k !}\right)\right|_{t^{d}} \prod_{i \geq 1}\left(1-q_{i}\right),
$$

with $\left.(-)\right|_{t^{d}}$ standing for the operation of taking the coefficient of $t^{d}$.

Thus we have the expansion

$$
\begin{aligned}
\operatorname{Hilb}_{\boldsymbol{q}}(\mathcal{C})=1+ & 2 q_{2} q_{1}+\left(3 q_{3} q_{1}+2 q_{2}{ }^{2}+3 q_{2} q_{1}{ }^{2}\right) \\
& +\left(4 q_{4} q_{1}+9 q_{3} q_{2}+6 q_{3} q_{1}{ }^{2}+10 q_{2}{ }^{2} q_{1}+4 q_{2} q_{1}{ }^{3}\right)+\ldots
\end{aligned}
$$

Corollary 2 will follow immediately from the explicit description of $\mathcal{C}$ and the isomorphism $\mathcal{A} \Lambda \rightarrow \mathcal{N}$ in Section 5, which is not only degree preserving, but shape preserving as well. 


\subsection{Algebra and coalgebra structures of $\mathcal{N}$}

Since the action of $\mathfrak{S}$ on $T$ is multiplicative, it is straightforward to see that $\mathcal{N}$ is an subalgebra of $T$. The multiplication rule in $\mathcal{N}$, expressing a product $m_{\mathbf{A}} \cdot m_{\mathbf{B}}$ as a sum of basis vectors $\sum_{\mathbf{C}} m_{\mathbf{C}}$, is easy to describe. Since we make heavy use of the rule later, we develop it carefully here. We begin with an example (the digits corresponding to $\mathbf{B}=1.2$ appear in bold):

$$
\begin{aligned}
m_{13.2} \cdot m_{1.2}= & m_{13.2 .4 .5}+m_{134.2 .5}+m_{135.2 .4} \\
& +m_{13.24 .5}+m_{13.25 .4}+m_{135.24}+m_{134.25}
\end{aligned}
$$

Compare this to (8). Notice that the shapes indexing the first and last terms in (13) are the partitions $\lambda(13.2) \cup \lambda(1.2)$ and $\lambda(13.2)+\lambda(1.2)$. As was the case in $S^{\mathfrak{S}}$, one of these shapes, namely $\lambda(\mathbf{A})+\lambda(\mathbf{B})$, will always appear in the product, while appearance of the shape $\lambda(\mathbf{A}) \cup \lambda(\mathbf{B})$ depends on the cardinality of $\mathbf{x}$.

Let us now describe the multiplication rule. Given any $D \subseteq \mathbb{N}$ and $k \in \mathbb{N}$, we write $D^{+k}$ for the set

$$
D^{+k}:=\{a+k \mid a \in D\} .
$$

By extension, for any set partition $\mathbf{A}=\left\{A_{1}, \ldots, A_{r}\right\}$ we set $\mathbf{A}^{+k}:=\left\{A_{1}^{+k}, A_{2}^{+k}, \ldots, A_{r}^{+k}\right\}$. These definitions allow for the introduction of a bilinear (non-commutative) operation denoted by " $\omega$ " on formal linear combinations of set partitions. Given partitions $\mathbf{A}=\left\{A_{1}, A_{2}, \ldots, A_{r}\right\}$ of $[c]$ and a partition $\mathbf{B}=\left\{B_{1}, B_{2}, \ldots, B_{s}\right\}$ of $[d]$, the summands of $\mathbf{A} w \mathbf{B}$ are set partitions of $[c+d]$. The operation $w$ is recursively defined by the rules:

(a) $\mathbf{A} w \emptyset=\emptyset \sim \mathbf{A}=\mathbf{A}$, with $\emptyset$ denoting the unique set partition of the empty set;

(b) $\mathbf{A} w \mathbf{B}=\left\{A_{1}\right\} \cup\left(\mathbf{A}^{\prime} w \mathbf{B}^{+c}\right)+\sum_{i=1}^{s}\left\{A_{1} \cup B_{i}^{+c}\right\} \cup\left(\mathbf{A}^{\prime} w\left(\mathbf{B} \backslash\left\{B_{i}\right\}\right)^{+c}\right)$,

with union $\cup$ extended bilinearly and $\mathbf{A}^{\prime}$ denoting $\left\{A_{2}, \ldots, A_{r}\right\}$.

As shown in [3, Prop. 3.2], the multiplication rule for $m_{\mathbf{A}}$ and $m_{\mathbf{B}}$ in $\mathcal{N}$, is

$$
m_{\mathbf{A}} \cdot m_{\mathbf{B}}=\sum_{\mathbf{C} \in \mathbf{A} w_{\mathbf{B}}} m_{\mathbf{C}}
$$

The subalgebra $\mathcal{N}$, like its commutative analog, is freely generated by certain monomial symmetric polynomials $\left\{m_{\mathbf{A}}\right\}_{\mathbf{A} \in \mathcal{A}}$, where $\mathcal{A}$ is some carefully chosen collection of set partitions. This is the main theorem of Wolf [14]. See also [3, §7]. We use two such collections later, our choice depending on whether or not $n<\infty$.

The operation $(-)^{+k}$ has a left inverse called the standardization operator and denoted by " $(-)^{\downarrow}$ ". It maps set partitions $\mathbf{A}$ of any cardinality- $d$ subset $D \subseteq \mathbb{N}$ to set partitions of $[d]$, with $\mathbf{A}^{\downarrow}$ defined as the pullback of $\mathbf{A}$ along the unique increasing bijection from $[d]$ to $D$. For example, $(18.4)^{\downarrow}=13.2$ and $(18.4 .67)^{\downarrow}=15.2 .34$. The coproduct $\Delta$ and counit $\varepsilon$ on $\mathcal{N}$ are given, respectively, by

$$
\Delta\left(m_{\mathbf{A}}\right)=\sum_{\mathbf{B} \cup \mathbf{C}=\mathbf{A}} m_{\mathbf{B} \downarrow} \otimes m_{\mathbf{C} \downarrow} \quad \text { and } \quad \varepsilon\left(m_{\mathbf{A}}\right)=\delta_{\mathbf{A}, \emptyset},
$$

where $\mathbf{B} \cup \mathbf{C}=\mathbf{A}$ means that $\mathbf{B}$ and $\mathbf{C}$ form complementary subsets of $\mathbf{A}$. In the case $n=\infty$, the maps $\Delta$ and $\varepsilon$ are algebra maps, making $\mathcal{N}$ a graded connected Hopf algebra. 


\section{The place-action of $\mathfrak{S}$ on $\mathcal{N}$}

\subsection{Swapping places in $T_{d}$ and $\mathcal{N}_{d}$}

On top of the permutation-action of the symmetric group $\mathfrak{S}_{\mathbf{x}}$ on $T$, we also consider the "place-action" of $\mathfrak{S}_{d}$ on the degree $d$ homogeneous component $T_{d}$. Observe that the permutation-action of $\sigma \in \mathfrak{S}_{\mathbf{x}}$ on a monomial $\mathbf{z}$ corresponds to the functional composition

$$
\sigma \circ \mathbf{z}:[d] \stackrel{\mathbf{z}}{\longrightarrow} \mathbf{x} \stackrel{\sigma}{\longrightarrow} \mathbf{x} .
$$

By contrast, the place-action of $\rho \in \mathfrak{S}_{d}$ on $\mathbf{z}$ gives the monomial

$$
\mathbf{z} \circ \rho:[d] \stackrel{\rho}{\longrightarrow}[d] \stackrel{\mathbf{z}}{\longrightarrow} \mathbf{x}
$$

composing $\rho$ with $\mathbf{z}$ on the right. In the linear extension of this action to all of $T_{d}$, it is easily seen that $\mathcal{N}_{d}$ (even each $\mathcal{N}_{\mu}$ ) is an invariant subspace of $T_{d}$. Indeed, for any set partition $\mathbf{A}=\left\{A_{1}, \ldots, A_{r}\right\} \vdash[d]$ and $\rho \in \mathfrak{S}_{d}$, one has (see $\left.[12, \S 2]\right)$

$$
m_{\mathbf{A}} \cdot \rho=m_{\rho^{-1} \cdot \mathbf{A}},
$$

where as usual $\rho^{-1} \cdot \mathbf{A}:=\left\{\rho^{-1}\left(A_{1}\right), \rho^{-1}\left(A_{2}\right), \ldots, \rho^{-1}\left(A_{r}\right)\right\}$.

\subsection{The place-action structure of $\mathcal{N}$}

Notice that the action in (15) is transitive on set partitions and is shape-preserving. It follows that a basis for the place-action invariants in $\mathcal{N}_{d}$ is indexed by partitions. For such a basis we choose the polynomials

$$
\mathbf{m}_{\mu}:=\frac{1}{\left(\operatorname{dim} \mathcal{N}_{\mu}\right) \mu^{!}} \sum_{\lambda(\mathbf{A})=\mu} m_{\mathbf{A}},
$$

with $\mu^{!}=a_{1} ! a_{2} ! \cdots$ whenever $\mu=1^{a_{1}} 2^{a_{2}} \ldots$. The normalizing coefficient will be explained in (19).

To simplify our discussion of the structure of $\mathcal{N}$ in this context, we will say that $\mathfrak{S}$ acts on $\mathcal{N}$ rather than being fastidious about underlying in each situation that individual $\mathcal{N}_{d}$ 's are being acted upon on the right by the corresponding group $\mathfrak{S}_{d}$. We also denote the set $\mathcal{N}^{\mathfrak{S}}$ of place-invariants by $\Lambda$. To summarize,

$$
\Lambda=\operatorname{span}\left\{\mathbf{m}_{\mu}: \mu \text { a partition of } d, d \in \mathbb{N}\right\} .
$$

The pair $(\mathcal{N}, \Lambda)$ begins to look like the pair $\left(S, S^{\mathfrak{S}}\right)$ from the introduction. This was the observation that originally motivated our search for Theorem 1 .

We next decompose $\mathcal{N}$ into irreducible place-action representations. Although this can be worked out for any value of $n$, the results are more elegant when we send $n$ to infinity. Recall that the Frobenius characteristic of a $\mathfrak{S}_{d}$-module $\mathcal{V}$ is the symmetric function

$$
\operatorname{Frob}(\mathcal{V})=\sum_{\mu \vdash d} v_{\mu} s_{\mu},
$$

where $s_{\mu}$ is a Schur function-the character of "the" irreducible $\mathfrak{S}_{d}$ representation $\mathcal{V}_{\mu}$ indexed by $\mu$-and $v_{\mu}$ is the multiplicity of $\mathcal{V}_{\mu}$ in $\mathcal{V}$. To reveal the $\mathfrak{S}_{d}$-module structure of $\mathcal{N}_{\mu}$ we may use (15) and standard techniques from the theory of combinatorial species, cf. [2]. The Frobenius characteristic of $\mathcal{N}_{\mu}$ is given by the following lemma. 
Lemma 3 For a partition $\mu=1^{a_{1}} 2^{a_{2}} \ldots k^{a_{k}}$, having $a_{i}$ parts of size $i$, we have

$$
\operatorname{Frob}\left(\mathcal{N}_{\mu}\right)=h_{d_{1}}\left[h_{1}\right] h_{d_{2}}\left[h_{2}\right] \cdots h_{d_{k}}\left[h_{k}\right],
$$

with $f[g]$ denoting plethysm of $f$ and $g$, and $h_{i}$ denoting the $i^{\text {th }}$ homogeneous symmetric function.

Recall that the plethysm $f[g]$ of two symmetric functions is obtained by linear and multiplicative extension of the rule $p_{k}\left[p_{\ell}\right]:=p_{k \ell}$, where the $p_{k}$ 's denote the usual power sum symmetric functions (see [10, I.8] for notations and more details). For instance, one finds that $h_{3}\left[h_{2}\right]=s_{6}+s_{42}+s_{222}$. That is, $\mathcal{N}_{222}$ decomposes into 3 irreducible components, with the trivial representation $s_{6}$ coming from $\mathbf{m}_{222}$ inside $\Lambda$.

\section{$4.3 \Lambda$ meets $S^{\mathfrak{S}}$}

We begin by explaining the choice of coefficient in (16). From [12, Thm. 2.1], one learns that the restriction to $\mathcal{N}$ of the abelianization map $\mathbf{a b}: T \rightarrow S$ (the map making the variables commute) satisfies:

(a) $\mathbf{a b}(\mathcal{N})=S^{\mathfrak{S}}$, and

(b) $\mathbf{a b}\left(m_{\mathbf{A}}\right)$ is a multiple of $m_{\lambda(\mathbf{A})}$ depending only on $\mu=\lambda(\mathbf{A})$, more precisely

$$
\mathbf{a b}\left(\mathbf{m}_{\mu}\right)=m_{\mu} .
$$

Formula (19) suggests that a natural right-inverse to $\mathbf{a b}(-)$ is given by

$$
\iota: S^{\mathfrak{S}} \hookrightarrow \mathcal{N}, \quad \text { with } \quad \iota\left(m_{\mu}\right):=\mathbf{m}_{\mu} .
$$

The fact that the image of $S^{\mathfrak{S}}$ in $\mathcal{N}$ is exactly the subspace $\Lambda$ affords us a quick proof of Theorem 1 in the case $n=\infty$. The isomorphism we construct for $n<\infty$ still uses the map $\iota$, but in a less essential way.

\section{The coinvariant space of $\mathcal{N}($ Case: $n=\infty)$}

\subsection{Proof of main result}

Suppose $n=\infty$. Combining results of [3] and a theorem of Blattner, Cohen, and Montgomery [6], we may immediately deduce the existence of a subspace $\mathcal{C}$ of $\mathcal{N}$ together with a vector space isomorphism $\mathcal{N} \simeq \mathcal{C} \otimes \Lambda$. Indeed, from Propositions 4.3 and 4.5 of [3], we get that the map $\iota$ is a coalgebra splitting of $\mathbf{a b}: \mathcal{N} \rightarrow S^{\mathfrak{S}} \rightarrow 0$, i.e.,

$$
\mathbf{a b} \circ \iota=\mathrm{id} \quad \text { and } \quad \Delta_{\mathcal{N}} \circ \iota=(\iota \otimes \iota) \circ \Delta_{S^{\mathfrak{S}}} \text {. }
$$

Moreover ab is a morphism of Hopf algebras. In this context, Theorem 4.14 of [6] suggests that we let $\mathcal{C}$ be the left Hopf kernel of the Hopf map ab,

$$
\mathcal{C}=\{h \in \mathcal{N}:(\text { id } \otimes \mathbf{a b}) \circ \Delta(h)=h \otimes 1\} .
$$

This theorem gives an algebra isomorphism between $\mathcal{N}$ and the crossed product $\mathcal{C} \#_{\sigma} S^{\mathfrak{S}}$. In fact, since $\Delta_{\mathcal{N}}$ is cocommutative, it is an isomorphism of Hopf algebras. We refer the interested reader to [6, §4] for the technical details. We mention only that: (i) the space $\mathcal{C}$ is actually a Hopf subalgebra of $\mathcal{N}$ by construction; (ii) the crossed product $\mathcal{C} \#_{\sigma} S^{\mathfrak{S}}$ is a certain algebra structure built on the tensor product $\mathcal{C} \otimes S^{\mathfrak{S}}$ using a cocyle $\sigma: S^{\mathfrak{S}} \times S^{\mathfrak{S}} \rightarrow \mathcal{C}$; and (iii) the isomorphism amounts to a cocyle twisting of simple multiplication: $\mathcal{C} \otimes S^{\mathfrak{S}} \mapsto \mathcal{C} \cdot \Lambda$. This completes the proof of Theorem 1. Moreover, since all spaces and morphisms are graded by degree, the Hilbert series for $\mathcal{C}$ is the quotient of that for $\mathcal{N}$ by that for $\Lambda$. This demonstrates (4). 


\subsection{Atomic set partitions.}

Recall the result of Wolf that $\mathcal{N}$ is a polynomial algebra, i.e., $\mathcal{N}$ is freely generated by some collection of polynomials. We announce our first choice for this collection now, following the terminology of [4]. Let $\Pi$ denote the set of all set partitions (of $[d], \forall d \geq 0$ ). We introduce the atomic set partitions $\dot{\Pi}$. A set partition $\mathbf{A}=\left\{A_{1}, \ldots, A_{r}\right\}$ of $[d]$ is atomic if there does not exist a pair $(s, c)(1 \leq s<r, 1 \leq c<d)$ such that $\left\{A_{1}, \ldots, A_{s}\right\}$ is a set partition of $[c]$. Conversely, $\mathbf{A}$ is not atomic if there are set partitions $\mathbf{B}$ of $\left[d^{\prime}\right]$ and $\mathbf{C}$ of $\left[d^{\prime \prime}\right]$ splitting $\mathbf{A}$ in two: $\mathbf{A}=\mathbf{B} \cup \mathbf{C}^{+d^{\prime}}$. We write $\mathbf{A}=\mathbf{B} \mathbf{C}$ in this situation. A maximal splitting $\mathbf{A}=\mathbf{A}^{\prime}\left|\mathbf{A}^{\prime \prime}\right| \cdots \mid \mathbf{A}^{(r)}$ of $\mathbf{A}$ is one where each $\mathbf{A}^{(i)}$ is atomic. For example, the partition 17.235.4.68 is atomic, while 12.346 .57 .8 is not. The maximal splitting of the latter would be $12|124.35| 1$, but we abuse notation and write $12|346.57| 8$ to improve legibility.

It is proven in [4] that $\mathcal{N}$ is freely generated by the atomic polynomials. To get a better sense of the structure, let us order $\Pi$ by giving $\dot{\Pi}$ a total order “ $\prec$ ” and then extending lexicographically. Given two atomic set partitions $\mathbf{A}$ and $\mathbf{B}$, we demand that $\mathbf{A} \prec \mathbf{B}$ if $\mathbf{A} \vdash[c]$ and $\mathbf{B} \vdash[d]$ with $c<d$. In case $\mathbf{A}, \mathbf{B}$ are partitions of the same set $[d]$, then any ordering will do for the current purpose... one interesting choice is to order $\mathbf{A}$ and $\mathbf{B}$ by ordering lexicographically their associated rhyme scheme words. ${ }^{\text {(ii) }}$ Our convention for writing set partitions provides a bijection between set partitions and this special class of words, sending $\mathbf{A}=\left\{A_{1}, A_{2}, \ldots, A_{r}\right\} \in \Pi_{d}$ to $w(\mathbf{A})=w_{1} w_{2} \cdots w_{d}$ defined by $w_{i}:=k$ if and only if $i \in A_{k}$. For example, $w(13.2)=121$ and $w(17.235 .4 .68)=12232414$. Using this ordering on $\dot{\Pi}$, we have the following chain within the set partitions of shape 3221 :

$$
1|23| 45|678 \prec 13.2| 456|78 \prec 13.24| 578.6 \prec 14.23 \mid 578.6 \prec 17.235 .4 .68 \prec 17.236 .4 .58 \text {. }
$$

In fact, $1|23| 45 \mid 678$ is the unique minimal element of $\Pi_{(3221)}$.

Define the leading term of a sum $\sum_{\mathbf{C}} \alpha_{\mathbf{C}} m_{\mathbf{C}}$ to be the monomial $m_{\mathbf{C}_{0}}$ such that $\mathbf{C}_{0}$ is lexicographically least among all $\mathbf{C}$ with $\alpha_{\mathbf{C}} \neq 0$. Combined with (14), our choice for $\prec$ makes it clear that the leading term of $m_{\mathbf{A}} \cdot m_{\mathbf{B}}$ is $m_{\mathbf{A} \mid \mathbf{B}}$. That is, multiplication in $\mathcal{N}$ is shape-filtered. Since the left Hopf kernel $\mathcal{C}$ is a subalgebra, it is shape-filtered as well. Finally, the isomorphism $\mathcal{C} \otimes \Lambda \rightarrow \mathcal{N}$ respects the shape structures on either side. This completes the proof of Corollary 2.

It is proven in [8] that $\mathcal{N}$ is not only freely generated by the atomic polynomials $\left\{m_{\mathbf{A}} \mid \mathbf{A} \in \dot{\Pi}\right\}$, but co-freely generated by them as well. By a classic theorem of Milnor and Moore [11], this means that $\mathcal{N}$ is isomorphic to the universal enveloping algebra $\mathfrak{U}(\mathfrak{L}(\dot{\Pi}))$ of the free Lie algebra $\mathfrak{L}(\dot{\Pi})$ on the set $\dot{\Pi}$. This description will be useful in the next subsection. Let us finish this section with a few final remarks on atomic set partitions. First, note that set partitions with one part are trivially atomic. The set of these is denoted by $\dot{\Pi}_{b}$. They are analogs of the generators $m_{k}$ for the algebra $S^{\mathfrak{S}}$. The remaining atomic set partitions

$$
\dot{\Pi}_{\sharp}:=\left\{\left\{A_{1}, \ldots, A_{r}\right\} \in \dot{\Pi}: r>1\right\}
$$

are more interesting. They index a large portion of the generators for $\mathcal{C}$. They are also the subject of an open question formulated at the end of Section 5.3.

\footnotetext{
(ii) Quoting Bill Blewett from [13, A000110], "a rhyme scheme is a string of letters (eg, abba) such that the leftmost letter is always $a$ and no letter may be greater than one more than the greatest letter to its left. Thus $a a c$ is not valid since $c$ is more than one greater than $a$. For example, $\left[\# \Pi_{3}=5\right.$ ] because there are 5 rhyme schemes on 3 letters: $a a a, a a b, a b a, a b b, a b c . "$
} 


\subsection{Explicit description of the Hopf algebra structure of $\mathcal{C}$}

It is not too hard to find elements in the left Hopf kernel of the abelianization map ab. Consider the following simple calculation. The sum of monomials $\tilde{m}_{13.2}:=m_{13.2}-m_{12.3}$ is primitive. Indeed,

$$
\begin{aligned}
\Delta\left(\tilde{m}_{13.2}\right)= & 1 \otimes m_{13.2}+m_{12} \otimes m_{1}+m_{1} \otimes m_{12}+m_{13.2} \otimes 1 \\
& -1 \otimes m_{12.3}-m_{12} \otimes m_{1}-m_{1} \otimes m_{12}-m_{12.3} \otimes 1 \\
= & 1 \otimes \tilde{m}_{13.2}+\tilde{m}_{13.2} \otimes 1 .
\end{aligned}
$$

We conclude that $($ id $\otimes \mathbf{a b}) \circ \Delta\left(\tilde{m}_{13.2}\right)=\tilde{m}_{13.2} \otimes 1$. In other terms, $\tilde{m}_{13.2} \in \mathcal{C}$. The linear map $\Delta$ may be split as $\Delta=\Delta^{\mathrm{P}}+\Delta^{\mathrm{I}}$, the sum of its primitive and imprimitive parts respectively. What we have just done in the example is to find a modification $\tilde{m}_{13.2}$ of $m_{13.2}$ satisfying $\Delta^{\mathrm{I}}\left(\tilde{m}_{13.2}\right)=0$. This suggests the following proposition.

Proposition 4 There is a primitive element

$$
\tilde{m}_{\mathbf{A}}=m_{\mathbf{A}}+\sum_{\mathbf{B}: \lambda(\mathbf{B})=\lambda(\mathbf{A})} \alpha_{\mathbf{B}} m_{\mathbf{B}}
$$

associated to each $\mathbf{A} \in \dot{\Pi}_{\sharp}$ such that $\sum_{\mathbf{B}} \alpha_{\mathbf{B}}=-1$ and $\mathbf{B} \in \dot{\Pi} \Rightarrow \alpha_{\mathbf{B}}=0$.

The existence of primitives comes from the Milnor-Moore isomorphism of $\mathcal{N}$ with $\mathfrak{U}(\mathfrak{L}(\dot{\Pi}))$. Showing that they can be chosen with the above properties is a simple calculation, inducting on the number of parts $r$ of an atomic set partition $\mathbf{A}=\left\{A_{1}, \ldots, A_{r}\right\}$ and applying $\left(\Delta^{\mathrm{I}}\right)^{r}$.

The ideas behind the proposition and the preceding example yield several immediate corollaries: (i) each $\tilde{m}_{\mathbf{A}}$ from Proposition 4 belongs to $\mathcal{C}$; (ii) $\mathcal{C}$ is shape-graded, i.e., if $h \in \mathcal{C}$ is written as $\sum_{\mu} h_{\mu}$, then each $h_{\mu}$ belongs to $\mathcal{C}$ as well; (iii) for any $g \in \mathcal{N}$ and $h \in \mathcal{C}$, we have that $[g, h]=g h-h g$ also belongs to $\mathcal{C}$; (iv) if $\mathbf{A}$ and $\mathbf{B}$ belong to $\dot{\Pi}_{b}$, then $\left[m_{\mathbf{A}}, m_{\mathbf{B}}\right]$ belongs to $\mathcal{C}$. These points essentially account for all of $\mathcal{C}$, as the next result suggests. First, recall that $S^{\mathfrak{S}}$ is also a universal enveloping algebra of a Lie algebra. Namely, the abelian Lie algebra $\mathfrak{A}\left(\left\{m_{1}, m_{2}, \ldots\right\}\right)$, where all Lie brackets $\left[m_{j}, m_{k}\right]$ are zero. Since the integers $k=1,2, \ldots$ are in $1-1$ correspondence with $\dot{\Pi}_{b}$, we have a natural map from $\mathfrak{L}(\dot{\Pi})$ to $\mathfrak{A}\left(\left\{m_{1}, m_{2} \ldots\right\}\right)$. Our final characterization of $\mathcal{C}$ is as follows.

Corollary 5 Let $\mathfrak{C}$ be the kernel of the map $\pi$ from the free Lie algebra on $\dot{\Pi}$ to the free abelian Lie algebra on $\dot{\Pi}_{b}$. Then the coinvariant space $\mathcal{C}$ is the universal enveloping algebra of the Lie algebra $\mathfrak{C}$.

Before turning to the case $n<\infty$, we remark that we have left unanswered the question of finding a systematic procedure (e.g., a closed formula in the spirit of Möbius inversion) that constructs a primitive element $\tilde{m}_{\mathbf{A}}$ for each $\mathbf{A} \in \dot{\Pi}_{\sharp}$.

\section{The coinvariant space of $\mathcal{N}$ (Case: $n<\infty)$}

We repeat our example of Section 3.3 in the case $n=3$. The leading term with respect to our previous order would be $m_{13.2 .4 .5}$, except that this term does not appear because 13.2.4.5 has more than $n=3$ parts. Fortunately, the rhyme scheme bijection $w$ reveals a more useful leading term:

$$
m_{121} \cdot m_{12}=0+m_{12113}+m_{12131}+m_{12123}+m_{12132}+m_{12121}+m_{12112} .
$$


The concatenation $121 \mid 12$ is the lexicographically smallest word appearing above. This is generally true: if $w(\mathbf{A})=u$ and $w(\mathbf{B})=v$, then $u v$ is the smallest element of $w(\mathbf{A} w \mathbf{B})$. Let us call a rhyme scheme word a verse if it cannot be written as the concatenation of two shorter rhyme schemes. The splitting of a rhyme scheme $w$ is the maximal deconcatenation $w=w^{\prime}\left|w^{\prime \prime}\right| \cdots \mid w^{(r)}$ of $w$ into verses $w^{(i)}$. For example, 12314 is a verse while 11232411 is a string of four versus $1|12324| 1 \mid 1$. It is easy to see that if $a, b, c$, and $d$ are verses, then $a|c=b| d$ if and only if $a=b$ and $c=d$. The preceding observations make it clear that $\mathcal{N}$ is verse-filtered and that $\mathcal{N}$ is freely generated by the monomials $\left\{m_{\boldsymbol{W}(\mathbf{A})} \mid \boldsymbol{w}(\mathbf{A})\right.$ is a verse $\}$. This is the collection of monomials originally chosen by Wolf, cf. [3, §7] for details.

Toward locating $\mathcal{C}$ within $\mathcal{N}$, we first locate $S^{\mathfrak{S}}$. Consider the partition $\mu=32211$. Note that the lexicographically least rhyme scheme word of shape $\mu$ is $w(123.45 .67 .8 .9)=111223345$. We are led to introduce the words

$$
w(\mu):=1^{\mu_{1}} 2^{\mu_{2}} \cdots k^{\mu_{k}}
$$

asociated to partitions $\mu=\left(\mu_{1}, \mu_{2}, \cdots, \mu_{k}\right)$; we call these descending rhymes since $\mu_{1} \geq \cdots \geq \mu_{k}$. Finally, we want to view $\mathcal{C}$ as the rhymes that don't involve a descending rhyme. Then, by the fact that $\mathcal{N}$ is verse-filtered, we will get an easy vector space isomorphism $\mathcal{C} \otimes \Lambda \rightarrow \mathcal{N}$ given by multiplication. Toward that end, we introduce the notion of vexillary rhymes.

A vexillary rhyme is a word that begins with a maximal (but possibly empty) descending rhyme, followed by one extra verse. The vexillary decomposition of a rhyme scheme $w$ is the expression of $w$ as a product $w=w^{\prime}\left|w^{\prime \prime}\right| \cdots\left|w^{(r)}\right| w^{(r+1)}$, where $w^{\prime}, \ldots, w^{(r)}$ are vexillary rhymes and $w^{(r+1)}$ is a possibly empty descending rhyme (which we call a tail). For a given word $w$, this decomposition is accomplished by first splitting $w$ into verses, then recombining, from left to right, consecutive verses to form vexillary rhymes. For instance, the splitting of 112212 is $1|1222| 12$. The first two factors combine to make one vexillary rhyme; the last factor is a descending tail: $1122212 \mapsto \overline{11222} \overline{12}$. Similarly,

$$
1231231411122311 \mapsto 123|12314| 1|1| 1223|1| 1 \mapsto \overline{12312314} \overline{111223} 11 .
$$

Suppose now that $u$ and $v$ are rhyme schemes and that the vexillary decomposition of $u$ is tail-free. Then by construction, the vexillary decomposition of $u v$ is the concatenation of the respective vexillary decompositions of $u$ and $v$. We are ready to identify $\mathcal{C}$ as a subalgebra of $\mathcal{N}$.

Theorem 6 Let $\mathcal{C}$ be the subalgebra of $\mathcal{N}$ generated by vexillary rhymes. Then $\mathcal{C}$ has a basis indexed by rhyme scheme words $w$ whose vexillary decompositions are tail-free. Moreover, the map $\mathcal{} \otimes \Lambda \rightarrow \mathcal{N}$ given by $m_{w^{\prime}} m_{w^{\prime \prime}} \cdots m_{w^{(r)}} \otimes m_{\left(\mu_{1} \cdots \mu_{k}\right)} \mapsto m_{w^{\prime}\left|w^{\prime \prime}\right| \cdots\left|w^{(r)}\right| \boldsymbol{W}(\mu)}$ is a vector space isomorphism.

\section{Other directions}

We conclude with another advertisement for the Blattner-Cohen-Montgomery theorem. The authors' present investigation into coinvariant spaces began by moving vertically within the commuting diagram (cube) of Hopf algebras depicted in Figure 1 (whereas in previous work, it was customary to move from left to right, cf. [1]). One may just as well move in other directions within the cube. To illustrate, we apply the Blattner-Cohen-Montgomery theorem to two other edges of interest (leaving aside any comments on group actions). The first of these concerns the downward arrow on the front-right side of the cube. Recall that, from a purely combinatorial perspective, bases in $\mathbb{K}\langle\mathbf{x}\rangle \sim \mathfrak{S}$ are indexed by "set compositions" (ordered set partitions), and those in $\mathbb{K}[\mathbf{x}]^{\sim \mathcal{S}}$ by integer compositions (here " $\sim$ " indicates the quasi-action 


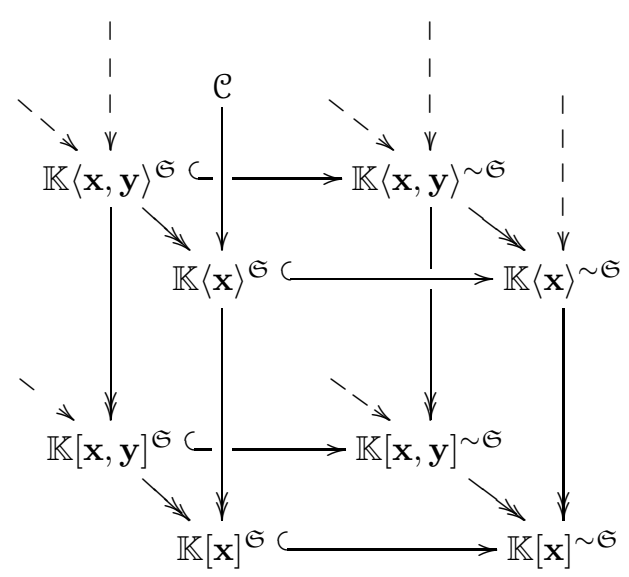

FIG. 1: The Hopf algebras of symmetric and quasisymmetric functions in one and two sets of commuting and noncommuting variables.

of Hivert, cf. [7, §3]). One may find a coalgebra splitting from $\mathbb{K}[\mathbf{x}]^{\sim \mathfrak{S}}$ to $\mathbb{K}\langle\mathbf{x}\rangle^{\sim \mathfrak{S}}$ and an associated coinvariant subalgebra in the spirit of our $\left(\mathcal{N}, S^{\mathfrak{S}}\right)$ investigation.

Another direction is to consider the Hopf algebra morphism sp : $\mathbb{K}[\mathbf{x}, \mathbf{y}]^{\sim \mathcal{S}} \rightarrow \mathbb{K}[\mathbf{x}]^{\sim \mathcal{S}}$ (the bottomright arrow going from NW to SE in Figure 1). These are the diagonally quasi-symmetric functions and quasi-symmetric functions respectively. For details omitted below, we refer the reader to [1]. The space $\mathbb{K}[\mathbf{x}, \mathbf{y}]^{\mathfrak{S}}$ is defined as the $\mathfrak{S}$-invariants, inside $\mathbb{K}[\mathbf{x}, \mathbf{y}]$, under the diagonal embedding of $\mathfrak{S}$ in $\mathfrak{S} \times \mathfrak{S}$. (The quasi-action of Hivert passes easily through this diagonal embedding.) A basis for $\mathbb{K}[\mathbf{x}, \mathbf{y}]^{\sim \mathfrak{S}}$ is given by the "monomial functions" $m_{\boldsymbol{a}, \boldsymbol{b}}$, indexed by "bicompositions", i.e., elements $(\boldsymbol{a}, \boldsymbol{b})$ in $\mathbb{N}^{2 \times r}$ such that $a_{i}+b_{i}>0$. These $m_{\boldsymbol{a}, \boldsymbol{b}}$ conveniently map to the quasi-symmetric function $m_{\boldsymbol{a}+\boldsymbol{b}}$ under the specialization map sp sending $y_{i}$ to $x_{i}$. It is straightforward to show that the map sending $m_{\boldsymbol{a}}$ to $m_{\boldsymbol{a}, \mathbf{0}}$, is a coalgebra splitting. We may thus analyze this situation in a manner analogous to our main result. Perhaps more surprising than the fact that the quotient

$$
\operatorname{Hilb}_{t}\left(\mathbb{K}[\mathbf{x}, \mathbf{y}]^{\sim \mathfrak{S}}\right) / \operatorname{Hilb}_{t}\left(\mathbb{K}[\mathbf{x}]^{\sim \mathfrak{S}}\right)
$$

belongs to $\mathbb{N} \llbracket t \rrbracket$ is the fact that the objects it counts have already been named. We discover a connection between compositions, set compositions, and "L-convex polyominoes." See [13, A003480].

\section{References}

[1] J.-C. Aval, F. Bergeron, and N. Bergeron. Diagonal Temperley-Lieb invariants and harmonics. Séminaire Lotharingien de Combinatoire, 54A (2005/07), Art. B54Aq, 19 pp. (electronic).

[2] F. Bergeron, G. Labelle, P. Leroux. Combinatorial Species and Tree-like Structures. Cambridge University Press, 1998.

[3] N. Bergeron, C. Reutenauer, M. Rosas, and M. Zabrocki. Invariants and coinvariants of the symmetric group in noncommuting variables. preprint, arXiv: math.CO/0502082. 
[4] N. Bergeron and M. Zabrocki. The Hopf algebras of symmetric functions and quasisymmetric functions in non-commutative variables are free and cofree. preprint, arXiv: math.CO/0509265.

[5] G. M. Bergman and P. M. Cohn. Symmetric elements in free powers of rings. J. London Math. Soc. (2), 1:525-534, 1969.

[6] R. J. Blattner, M. Cohen, and S. Montgomery. Crossed products and inner actions of Hopf algebras. Trans. Amer. Math. Soc., 298(2):671-711, 1986.

[7] F. Hivert. Hecke algebras, difference operators, and quasi-symmetric functions. Adv. Math., 155(2):181-238, 2000.

[8] F. Hivert, J.-C.Novelli, and J.-Y. Thibon. Commutative Hopf algebras of permutations and trees. preprint, arXiv: math.CO/0502456.

[9] M. Klazar, Bell numbers, their relatives, and algebraic differential equations, J. Combin. Theory Ser. A, 102(1):63-87, 2003.

[10] I. G. Macdonald, Symmetric functions and Hall polynomials, second ed., Oxford Mathematical Monographs. The Clarendon Press Oxford University Press, New York, 1995, With contributions by A. Zelevinsky, Oxford Science Publications.

[11] J. W. Milnor and J. C. Moore. On the structure of Hopf algebras. Ann. of Math. (2), 81:211-264, 1965.

[12] M. H. Rosas and B. E. Sagan. Symmetric functions in noncommuting variables. Trans. Amer. Math. Soc., 358(1):215-232 (electronic), 2006.

[13] N. J. A. Sloane. The On-Line Encyclopedia of Integer Sequences (2007). published electronically at www.research.att.com/njas/sequences/.

[14] M. C. Wolf. Symmetric functions of non-commutative elements. Duke Math. J., 2(4):626-637, 1936. 
